\title{
OPEN AND MONOTONE FIXED POINT FREE MAPS ON UNIQUELY ARCWISE CONNECTED CONTINUA
}

\author{
LEE MOHLER AND LEX G. OVERSTEEGEN ${ }^{1}$
}

\begin{abstract}
In this note we will construct uniquely arcwise connected continua admitting open and monotone fixed point free mappings, respectively. We will also show that each locally one-to-one map on a uniquely arcwise connected continuum has a fixed point.
\end{abstract}

Introduction. A continuum is a compact and connected metric space. A continuum $X$ is said to be uniquely arcwise connected (u.a.c) if given any two points $x, y \in X$, $x \neq y$, there is a unique arc in $X$ whose endpoints are $x$ and $y$. This is equivalent to saying that $X$ is arcwise connected and contains no simple closed curves. It is known [5] that u.a.c. need not have the fixed point property for continuous mappings but [3] they do have the fixed point property for homeomorphisms.

A continuum $X$ is called decomposable provided there exist two proper subcontinua $A, B \subset X$ such that $X=A \cup B$ and hereditarily decomposable provided each subcontinuum is decomposable. A continuum is called indecomposable provided it is not decomposable. A map $f: X \rightarrow Y$ is called open provided $f(U)$ is open for each open set $U \subset X$ and monotone provided $f^{-1}(y)$ is connected for each $y \in Y$. A map $f: X \rightarrow Y$ is open on $A \subset X$ provided, for each $x \in A$, there exists an open set $U$ containing $x$ such that $f(U)$ is open. A map $f: X \rightarrow Y$ is called locally one-to-one provided that for each $x \in X$ there exists an open set $U \subset X$ containing $x$ such that $f \mid U$ is one-to-one. We will show that each locally one-to-one map $f: X \rightarrow X$ where $X$ is a u.a.c. continuum has a fixed point. We will also show that this result is the best possible by constructing hereditarily decomposable u.a.c. continua admitting monotone and open fixed point free maps, respectively. It follows from a result of Hagopian [2] that these examples are nonplanar.

If $x$ and $y$ are points in a u.a.c. continuum $X$, we denote by $[x, y]$ the unique irreducible arc joining them. Let $X$ be a continuum and let $x \in X$. By $o(x, X)$ we denote the minimal cardinal number $\alpha$ with the property: For each open set $U \subset X$ containing $x$, there exists an open set $V, x \in V \subset U$, such that $|\operatorname{Bd}(V)| \leqslant \alpha$. We let $\omega$ denote the first infinite cardinal number. We will make use of the following observation:

Lemma 0 . Let $Z$ be an indecomposable subcontinuum of a continuum $X$ and let $z \in Z$. Then $o(z, X)>\omega$.

Received by the editors April 20, 1984 and, in revised form, December 11, 1984.

The results of this paper were announced at the AMS Summer Meeting in Bellingham, Washington, 1982.

1980 Mathematics Subject Classification. Primary 54F20; Secondary 54F50.

${ }^{1}$ The second author was supported in part by NSF grant number MCS- 8104866 .

(c)1985 American Mathematical Society $0002-9939 / 85 \$ 1.00+\$ .25$ per page 
THEOREM 1. Let $X$ be a u.a.c. continuum and let $f: X \rightarrow X$ be a locally one-to-one map. Then $f$ has a fixed point.

Proof. Suppose $Y_{0}=f(X) \nsubseteq X$. Define $Y_{\alpha}$ by transfinite induction as follows: $Y_{\alpha+1}=f\left(Y_{\alpha}\right)$ and $Y_{\gamma}=\bigcap_{\alpha<\gamma} Y_{\alpha}$ if $\gamma$ is a limit ordinal. Then $Y_{\alpha} \subset Y_{\beta}$ if $\alpha<\beta$. It follows easily (using transfinite induction) that $Y_{\alpha}$ is uniquely arcwise connected for each $\alpha$. Since $X$ is a continuum, there exists an index $\alpha_{0}$ such that $Y_{\alpha_{0}}=Y_{\alpha_{0}+1}$. Then $f \mid Y_{\alpha_{0}}: Y_{\alpha_{0}} \rightarrow Y_{\alpha_{0}}$ is a locally one-to-one map from the u.a.c. continuum $Y_{\alpha_{0}}$ onto $Y_{\alpha_{0}}$. We claim that $f$ is one-to-one. To this end, suppose there exist $x_{1} \neq x_{2} \in X$ such that $f\left(x_{1}\right)=f\left(x_{2}\right)$. Let $A$ be an arc joining $x_{1}$ and $x_{2}$, let $<$ be an order on $A$ and let 0 denote the minimum of $A$. Let $z_{1}=\min \{a \in A \mid f(a) \in f([0, a))\}$, and let $z_{0}=$ $\max \left\{a \in\left[0, z_{1}\right) \mid f(a)=f\left(z_{1}\right)\right\}$. Then $z_{0}<z_{1}$ and $f\left(\left[z_{0}, z_{1}\right]\right)$ is a simple closed curve. This contradicts the fact that $X$ is uniquely arcwise connected. Hence, $f \mid Y_{\alpha_{0}}$ : $Y_{\alpha_{0}} \rightarrow Y_{\alpha_{0}}$ is a homeomorphism. It follows from [3] that $f \mid Y_{\alpha_{0}}$ has a fixed point and the proof of the theorem is complete.

Recall that Young's example [5] of a fixed point free map on a uniquely arcwise connected continuum $X$ consists of a double Warsaw circle $W$ (see Figure 1 ) in the $x y$-plane in $\mathbf{R}^{3}$, an arc joining the points $a=(0,-1,0)$ and $b=(0,1,0)$ and a ray $R$ with initial point $v=(0,0,0)$ which compactifies onto $W$.

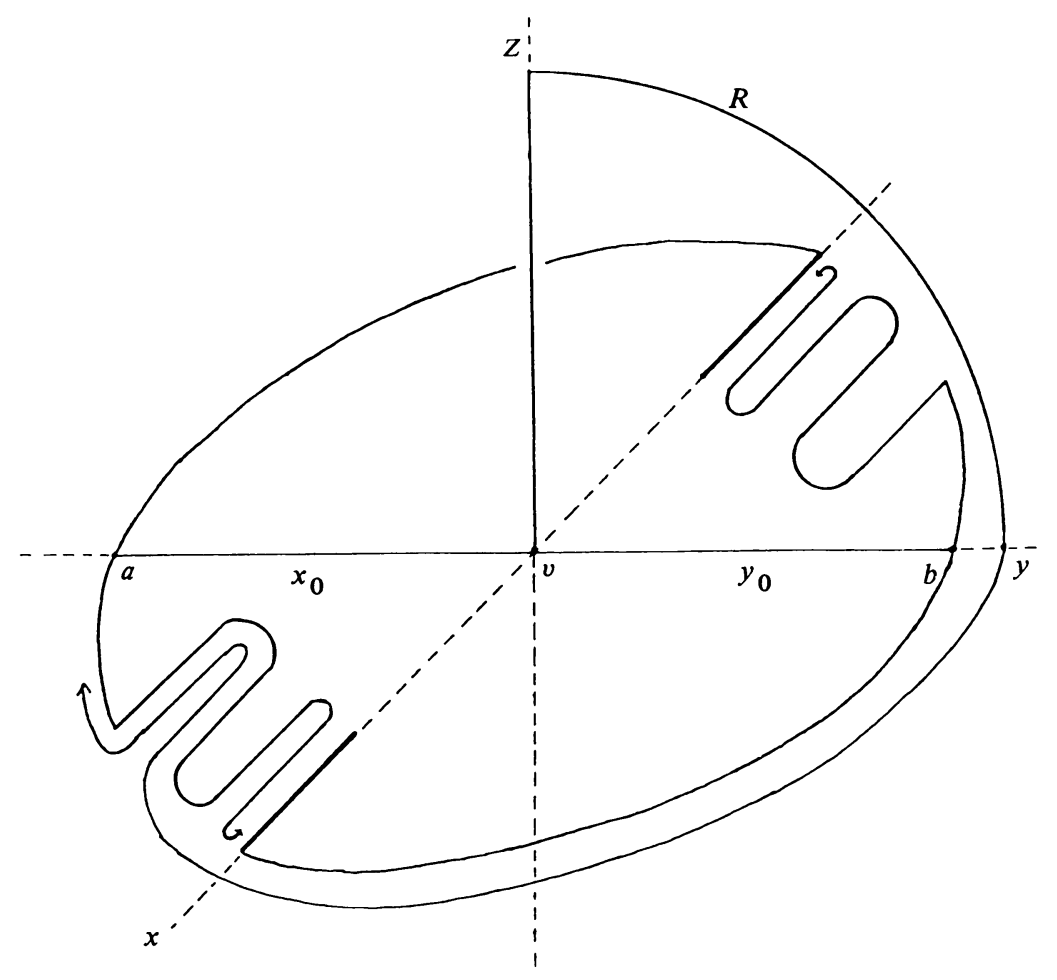

FIGURE 1 
The fixed point free map $f: X \rightarrow X$ rotates $W=180^{\circ}$, interchanging the points $a$ and $b$, maps $\left[a, x_{0}\right]$ onto $[b, v]$ and $\left[b, y_{0}\right]$ onto $[a, v]$ and maps the ray $R$ into itself such that $f|R, f|\left[x_{0}, v\right]$ and $f \mid\left[y_{0}, v\right]$ are one-to-one. It follows that $f$ is open on $X \backslash\left\{x_{0}, y_{0}\right\}$ and $f \mid X \backslash\left[x_{0}, y_{0}\right]$ is one-to-one (and, in particular, monotone). We will assume that $f(0, y, 0)=(0,-1-2 y, 0)$ for each $y \in\left[a, x_{0}\right]$ and $f(0, y, 0)=$ $(0,1-2 y, 0)$ for each $y \in\left[y_{0}, b\right]$.

EXAMPLE 1. There exists a hereditarily decomposable u.a.c. continuum $Z$ admitting a monotone fixed point free map $g: Z \rightarrow Z$.

Proof. Let $X$ denote Young's example and let $f: X \rightarrow X$ be the fixed point free map described above. Note that $f \mid X \backslash\left[x_{0}, y_{0}\right]$ is one-to-one and hence monotone. We will connect the points $(0, y, 0)$ and $(0,-y, 0)$ on $[a, b]$ with a continuum such that $f$ admits a monotone fixed point free extension. Let $C \subset I$ be the standard Cantor ternary set in $[0,1]$ obtained by removing the "middle third" intervals. Let $h$ : $I \rightarrow I$ be a monotone map such that $h(C)=I$ and $h \mid C$ is the "standard" Lebesgue singular function (i.e. $h\left(\frac{1}{3}\right)=h\left(\frac{2}{3}\right)=\frac{1}{2}, h\left(\frac{1}{9}\right)=h\left(\frac{2}{9}\right)=\frac{1}{4}, \ldots$ ). Let $W$ be the double Warsaw circle described above and consider $W \times C$. Let $(W \times C)^{*}=$ $(W \times C) /(W \times\{1\})$ and let $\phi: W \times\{0\} \rightarrow W \subset X$ denote the natural homeomorphism. Let $Z$ be obtained from the disjoint union of $(W \times C)^{*}$ and $X$ by identifying each point of $(w, 0)$ with $\phi((w, 0))$, each point $(a, c)$ with $(0, h(c)-1,0) \in X$ and each point $(b, c)$ with $(0,1-h(c), 0)$. Then $Z$ is a u.a.c. continuum. Each pair of points $(0, y, 0)$ and $(0,-y, 0)$ where $y$ is not of the form $k / 2^{n}$ are joined by a homeomorphic copy $W_{y}$ of $W$ and each pair of points $(0, y, 0)$ and $(0,-y, 0)$ where $y$ is of the form $k / 2^{n}$ are joined by a continuum $W_{y}$ which is obtained from two homeomorphic copies of $W$ by identifying the points corresponding to $a$ and $b$, respectively. Define the fixed point free extension $g: Z \rightarrow Z$ such that $g\left(W_{y}\right)=$ $f(0, y, 0) \in R$ if $|y|<\frac{1}{2}, g \mid W_{y}: W_{y} \rightarrow W_{-2 y-1},-1 \leqslant y \leqslant-\frac{1}{2}$, and $g \mid W_{y}: W_{y} \rightarrow$ $W_{-2 y+1}, \frac{1}{2} \leqslant y \leqslant 1$, are the natural homeomorphism followed by a $180^{\circ}$ rotation. It is not difficult to see that $g$ is the required fixed point free extension of $f$. Note that if $x \in X \backslash[a, b] \subset Z$, then $o(x, X)=\omega$. It is not difficult to see that $[a, b] \cup Z \backslash X$ is hereditarily decomposable. By Lemma $0, Z$ is hereditarily decomposable.

We will next construct a hereditarily decomposable u.a.c. $X$ admitting an open fixed point free map. We start out with some preliminaries. By a $\lambda$-dendroid $D$ we mean a hereditarily decomposable, hereditarily unicoherent continuum. Note that for each pair of points in $D$, there is at most one arc joining them. A dendroid is an arcwise connected $\lambda$-dendroid.

EXAMPLE 2 . There exists a $\lambda$-dendroid $D$ admitting an open and monotone map $\phi$ : $D \rightarrow[0,1]$ such that $\phi^{-1}(x)$ is a point for each $x \in\left[0, \frac{3}{4}\right] \cup\left[\frac{7}{8}, 1\right]$ and $D$ contains exactly two arc components $A_{1}$ and $A_{2}$ such that $f^{-1}(0) \in A_{1}$ and $f^{-1}(1) \in A_{2}$.

Proof. In [4], the second author constructed a dendroid $Z$ admitting a monotone and open retraction $r: Z \rightarrow J=[0,1]$ where $J$ is an arc such that $r^{-1}(t)$ is nondegenerate for each $t \in J$. Let $Z^{*}=Z / r^{-1}(0)$, let $\pi: Z \rightarrow Z^{*}$ be the natural projection and let $r^{*}: Z^{*} \rightarrow[0,1]$ be defined by $r^{*}(z)=r(z)$ if $z \in Z \backslash r^{-1}(0) \subset Z^{*}$ and $r^{*}\left(\pi \circ\left\{r^{-1}(0)\right\}\right)=0$. Then $r^{*}$ is an open and monotone retraction of the dendroid $Z^{*}$ onto $[0,1]$ such that $r^{*-1}(t)$ is nondegenerate for each $t \in(0,1]$. Let 
$Z^{-}=r^{*-1}([0,1)), J^{*}=\pi(J)$, let $J^{-}=J^{*} \backslash\{1\} \subset Z^{-}$and let $B$ be a homeomorphic copy of $r^{*-1}(1) \subset Z^{*}$. Then $J^{-}$is a ray. Let $Z^{+}$be a compactification of $Z^{-}$with remainder $B$ such that $Z^{-} \cap B=\varnothing, \mathrm{Cl}\left(J^{-}\right) \backslash J^{-}=B$. Moreover, since $r^{*-1}(t)$ is nondegenerate (and has diameter greater than some number $\varepsilon>0$ ), we can choose the compactification such that $\operatorname{Lim} r^{*-1}\left(t_{n}\right)=B$ for each sequence $t_{n} \in J^{\text {- such that }}$ $\lim \left(t_{n}\right)=\{1\}$. It follows that there exists an open and monotone map $r^{+}: Z^{+} \rightarrow[0,1]$ defined by $r^{+}(z)=r^{*}(z)$ if $z \in Z^{-} \subset Z^{+}$and $r^{+}(z)=1$ if $z \in B$. Moreover, $Z^{+}$is a $\lambda$-dendroid which contains exactly two arc components, namely $Z^{-}$and $B$. Let $D$ be the $\lambda$-dendroid obtained from the disjoint union of $Z^{*}, Z^{+}$and the arcs $[-2,-1]$ and $[1,2]$ by identifying $r^{*-1}(1) \subset Z^{*}$ with $B \subset Z^{+}$under the natural homeomorphism, the point $\left(r^{+}\right)^{-1}(0)$ with -1 and the point $r^{*-1}(0)$ with 1 . It is now not difficult to construct the required map $\phi: D \rightarrow[0,1]$. We will picture the $\lambda$-dendroid $D$ as in Figure 2 and label the point $\phi^{-1}(0)$ and $\phi^{-1}(1), a$ and $b$, respectively.

It is not difficult to see that the "double Warsaw" circle in Young's example can be replaced by any u.a.c. containing exactly two arc components which admits a $180^{\circ}$ rotation. We will replace (see Figure 3 ) $W$ by the disjoint union of two homeomorphic copies, $D^{+}$and $D^{-}$of $D$ where the point corresponding to $a(b)$ in $D^{+}$is identified with the point corresponding to $b$ ( $a$, respectively) in $D_{-}$. We will label the first point by $\alpha$ and the second by $\beta$.

Let $X_{1}$ denote this modified Young example and let $f_{1}: X_{1} \rightarrow X_{1}$ be the corresponding fixed point free map. As indicated above, $f_{1}$ is open on $X \backslash f_{1}^{-1}(v)$. Put $f_{1}^{-1}(v)=V_{1}=\left\{x_{0}, y_{0}\right\}$.

The required u.a.c. continuum $X$ will be constructed as an inverse limit. Let $X$ $=\lim \left(X_{n}, r_{m}^{n}\right)$ where each map $r_{m}^{n}: X_{n} \rightarrow X_{m}$ is a retraction. We will assume that $X_{m} \leftarrow X_{n} \subset X$ for each $n \leqslant m$ and denote by $r_{n}: X \rightarrow X_{n}$ the natural projection. Note that $r_{n}$ is a retraction.

Lemma 2. Let $X=\lim \left(X_{n}, r_{m}^{n}\right)$, where $r_{m}^{n}: X_{n} \rightarrow X_{m}$ is a retraction and $X_{n}$ is a u.a.c. continuum for each $\tilde{n} \geqslant 1$. Suppose that for each $x \in X$, there exists $n_{0} \geqslant 1$ such that $r_{n}(x)=r_{n_{0}}(x)$ for all $n \geqslant n_{0}$. Then $x$ is a u.a.c. continuum and $X$ is homeomorphic with $\cup_{n} X_{n}$. We will identify $X$ with $\cup_{n} X_{n}$. Hence for each $x \in X$ there exists $n$ such that $r_{n}(x)=x$.

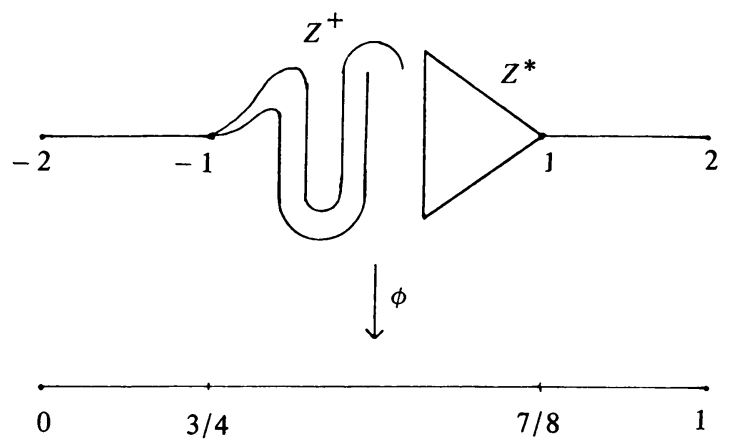

FIGURE 2 
Proof. Suppose $x, y \in X$. Let $n$ be so large that $x=r_{n}(x)$ and $y=r_{n}(y)$. Since $X_{n}$ is u.a.c., there exists an arc $J \subset X_{n} \subset X$ joining $x$ and $y$. Hence $X$ is arcwise connected. Suppose next that $S \subset X$ is a simple closed curve. Then there exists $n$ such that $r_{n}(S) \subset X_{n}$ contains a simple closed curve. This contradicts the fact that $X_{n}$ is u.a.c. and completes the proof.

If $X$ is a continuum and $a, b \in A \subset X$, we say that $Y$ is obtained from $X$ by doubling $A \backslash\{a, b\}$ provided $Y$ is homeomorphic to the space obtained from the disjoint union of $X$ and a disjoint copy of $A$ by identifying the points corresponding to $a$ and $b$, respectively. We will always assume that $X \subset Y$. Hence there exists a natural retraction $r: Y \rightarrow X$.

EXAMPLE 3 . There exists a hereditarily decomposable uniquely arcwise connected continuum $X$ admitting an open fixed point free map $f: X \rightarrow X$.

Proof. Let $X_{1}$ be the modified Young example (see Figure 3) constructed above and let $f_{1}: X_{1} \rightarrow X_{1}$ denote the corresponding fixed point free map. Put $V_{1}=f_{1}^{-1}(v)$ $=\left\{x_{0}, y_{0}\right\}\left(x_{0}=\left(0,-\frac{1}{2}, 0\right), y_{0}=\left(0, \frac{1}{2}, 0\right) \in \mathbf{R}^{3}\right)$. Then $f_{1}$ is open on $X_{1} \backslash\left\{x_{0}, y_{0}\right\}$. Recall that there exists an open map $\phi: D \rightarrow[0,1]$ (see Example 2). Let $X_{2}$ (see Figure 4$)$ be the space obtained from $X_{1}$ by doubling $\left[\alpha, x_{0}\right] \cup D^{-} \backslash\left\{x_{0}, \beta\right\}$ and $\left[y_{0}, \beta\right] \cup D^{+} \backslash\left\{y_{0}, \alpha\right\}$.

Let $r_{1}^{2}: X_{2} \rightarrow X_{1}$ be the natural retraction and let $f_{2}: X_{2} \rightarrow X_{1}$ be an extension of $f_{1}$ such that $f_{2}$ maps the attached copy of $\left[x_{0}, \alpha\right] \cup D^{-}\left(\left[y_{0}, \beta\right] \cup D^{+}\right)$open onto $[\beta, v]$ $\left([\alpha, v]\right.$, respectively) such that $f_{2}^{-1}(t)$ consists of exactly two points for each $t \in$ $\left[\alpha, x_{0}\right] \cup\left[\beta, y_{0}\right]$. Note that $f_{2}$ is open on the set $X_{2} \backslash f_{2}^{-1}\left(\left\{x_{0}, y_{0}\right\}\right) \cup\{\alpha, \beta\}$ and that $r_{1}^{2} \circ f_{2}: X_{2} \rightarrow X_{1}$ is open. Put $f_{2}^{-1}\left(x_{0}\right)=\left\{y_{1}, y_{2}\right\}=A_{1}$ and $f_{2}^{-1}\left(y_{0}\right)=\left\{x_{1}, x_{2}\right\}$ $=B_{1}$. Let $X_{3}$ be the space obtained from $X_{2}$ by doubling $\left[x_{1}, \alpha\right] \cup$ $D^{-} \backslash\left\{x_{1}, \beta\right\},\left[x_{2}, \alpha\right] \cup D^{-} \backslash\left\{x_{2}, \beta\right\},\left[y_{1}, \beta\right] \cup D^{+} \backslash\left\{y_{1}, \alpha\right\}$ and $\left[y_{2}, \beta\right] \cup D^{+} \backslash\left\{y_{2}, \alpha\right\}$ and let $r_{2}^{3}: X_{3} \rightarrow X_{2}$ be the natural retraction. Let $f_{3}: X_{3} \rightarrow X_{3}$ be an extension of $f_{2}$ such that $f_{3}$ restricted to the closure of each component of $X_{3} \backslash X_{2}$ is one-to-one. Put

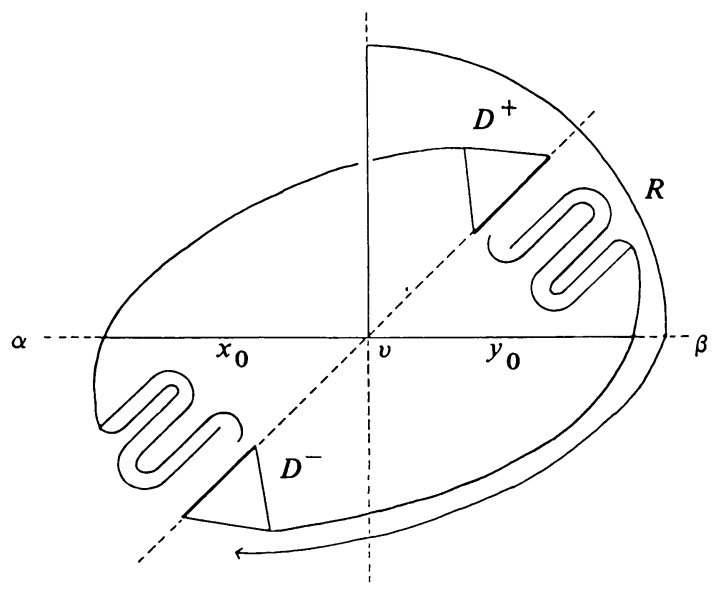

FIGURE 3 


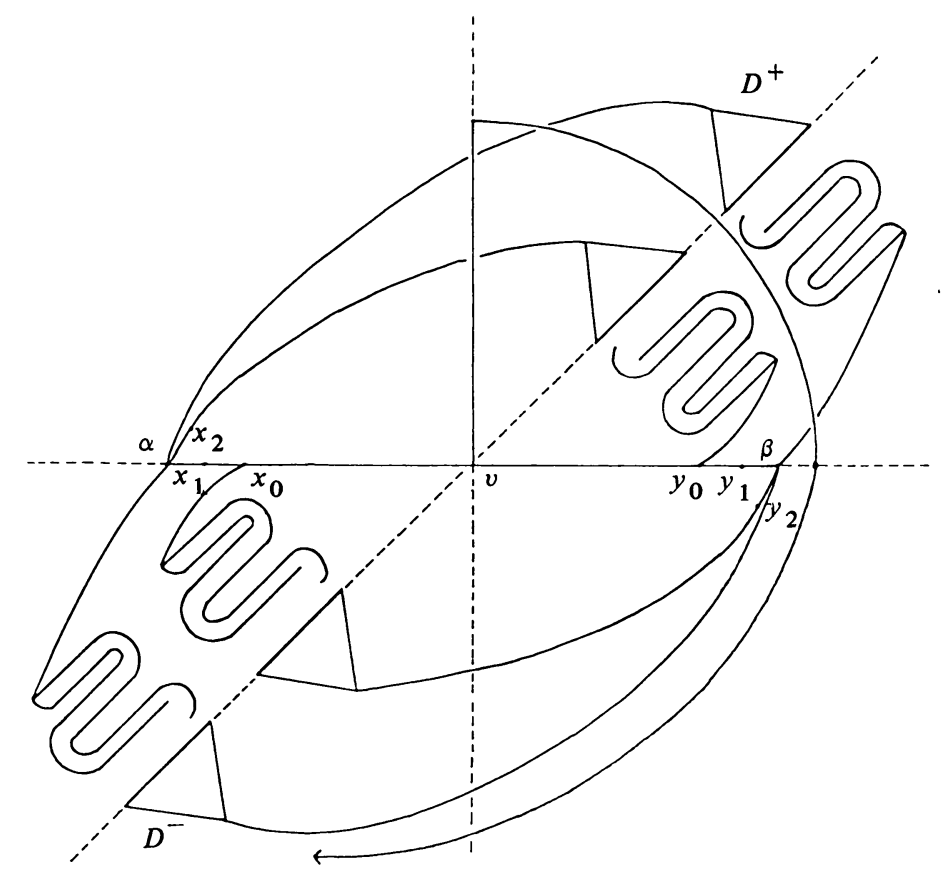

FIGURE 4

$V_{3}=f_{3}^{-1} \circ f_{3}^{-1} \circ f_{3}^{-1}(v)=f_{3}^{-1}\left\{x_{1}, x_{2}, y_{1}, y_{2}\right\}$, then $f_{3}$ is open on the set $X \backslash V_{3} \cup\{\alpha, \beta\}$ and $r_{2}^{3} \circ f_{3}: X_{3} \rightarrow X_{2}$ is open. Note that, for each point $x \in V_{3}$, $f_{3}^{-1}(x)$ consists of exactly two points. Put $A_{2}=f_{3}^{-1}\left(A_{1}\right)$ and $B_{2}=f_{3}^{-1}\left(B_{1}\right)$.

Suppose $X_{n}, f_{n}, r_{m}^{n}, A_{n-1}$ and $B_{n-1}$ have been constructed for $m \leqslant n \leqslant n_{0}$ such that $f_{n_{0}}$ is open on the set $X_{n_{0}} \backslash A_{n-1} \cup B_{n-1} \cup\{\alpha, \beta\}$. Let $X_{n_{0}+1}$ be obtained from $X_{n_{0}}$ by doubling $[\alpha, a] \cup D^{-} \backslash\{\alpha, \beta\}$ for each $a \in A_{n-1}$ and $[b, \beta] \cup D^{+} \backslash\{b, \alpha\}$ for each $b \in B_{n-1}$ and let $r_{n_{0}}^{n_{0}+1}: X_{n_{0}+1} \rightarrow X_{n_{0}}$ be the natural projection. Let $f_{n_{0}+1}$ : $X_{n_{0}+1} \rightarrow X_{n_{0}+1}$ be an extension of $f_{n_{0}}$ such that $f_{n_{0}+1}$ restricted to the closure of each component of $X_{n_{0}+1} \backslash X_{n_{0}}$ is one-to-one. If $C$ is the closure of a component of $X_{n_{0}+1} \backslash X_{n_{0}}$, then $f_{n_{0}+1}(C)=C^{\prime}$ is the closure of a component of $X_{n_{0}} \backslash X_{n_{0}-1}$. We will define $f_{n_{0}+1}$ such that

$$
r_{n_{0}-1}^{n_{0}+1} \circ f_{n_{0}+1}\left|C=r_{n_{0}-1}^{n_{0}} \circ f_{n_{0}} \circ r_{n_{0}}^{n_{0}+1}\right| C .
$$

Put $A_{n_{0}}=f_{n_{0}+1}^{-1}\left(A_{n_{0}-1}\right)$ and $B_{n_{0}}=f_{n_{0}+1}^{-1}\left(B_{n_{0}-1}\right)$. Then $f_{n_{0}+1}$ is open on the set $X_{n_{0}+1} \backslash A_{n_{0}} \cup B_{n_{0}} \cup\{\alpha, \beta\}$ and $r_{n_{0}}^{n_{0}+1} \circ f_{n_{0}+1}: X_{n_{0}+1} \rightarrow X_{n_{0}}$ is open. Let $X$ $=\lim _{\leftarrow}\left(X_{n}, r_{m}^{n}\right)$. By Lemma $2, X$ is a u.a.c. continuum. It follows from (1) that the sequence $r_{n-1}^{n} \circ f_{n}: X_{n} \rightarrow X_{n-1}$ induces an open map $f: X \rightarrow X$. It is not difficult to check that $f$ is the required fixed point free map. It is not difficult to see that if $Q=\{x \in X \mid o(x, X) \leqslant \omega\}$ (note that $\alpha, \beta \in Q)$ and if $Z \subset X \backslash Q$ is a continuum, then $Z$ is a subset of a homeomorphic copy of the $\lambda$-dendroid $D$ constructed in Example 2. (Recall that the retractions $r_{n}^{n+1}$ used in the inverse limit description of $X$, are "nondegenerate" essentially only on the sets $D^{-} \cup D^{+}$.) By Lemma $0, X$ is hereditarily decomposable. 
REMARK. A. Conner has announced the existence of an open and monotone fixed point free map on a u.a.c. continuum. This example is obtained from the hyperspace of subcontinua $C(P)$ of the pseudo-circle $P$ to which a ray $R$ is added starting at the point $\{P\} \in C(P)$ which compactifies on the "base" $P \subset C(P)$. The following problem remains open.

Problem 1. Does there exist an hereditarily decomposable u.a.c. continuum which admits an open and monotone fixed point free map?

\section{REFERENCES}

1. A. Conner (to appear).

2. C. L. Hagopian, Uniquely arcwise connected plane continua have the fixed point property, Trans. Amer. Math. Soc. 248 (1979), 85-104.

3. L. Mohler, The fixed point property for homeomorphisms of 1-arcwise connected continua, Proc. Amer. Math. Soc. 25 (1975), 451-456.

4. L. G. Oversteegen, Open retractions and locally confluent mappings of certain continua, Houston $\mathrm{J}$. Math. 6 (1980), 113-125.

5. G. G. Young, Jr., Fixed point theorems for arcwise connected continua, Proc. Amer. Math. Soc. 11 (1960), 880-884.

Department of Mathematics, University of Alabama in Birmingham, Birmingham, Alabama 35294 